\title{
DNFB activates MAPKs and upregulates CD40 in skin-derived dendritic cells
}

\author{
Teresa J. Matos a , Carlos B. Duarte ${ }^{a, b, *}$, Margarida Gonçalo ${ }^{c}$, \\ M. Celeste Lopes ${ }^{\text {a,d }}$
}
${ }^{a}$ Centro de Neurosciências e Biologia Celular, Universidade de Coimbra, 3004-517 Coimbra, Portugal
${ }^{\mathrm{b}}$ Departamento de Zoologia, Universidade de Coimbra, Portugal
' Faculdade de Medicina (Serviço de Dermatologia), Hospital da Universidade
de Coimbra, 3000-075 Coimbra, Portugal
${ }^{\mathrm{d}}$ Faculdade de Farmácia, Universidade de Coimbra, Rua do Norte, 3000-295 Coimbra, Portugal

Received 20 December 2004; received in revised form 28 March 2005; accepted 31 March 2005

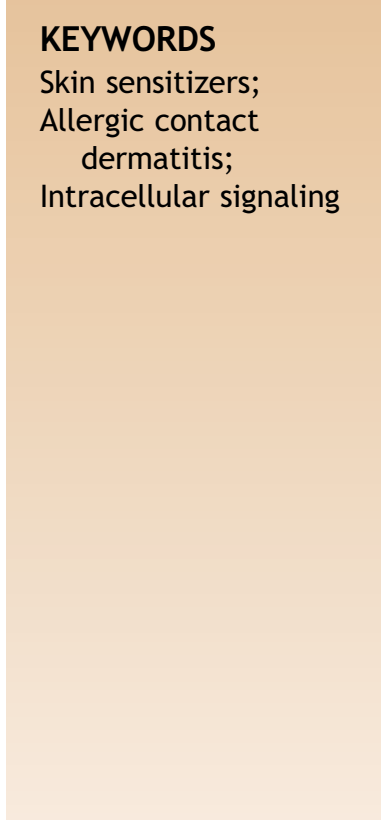

\begin{abstract}
Summary
Background: The intracellular mechanisms involved in the activation of DCs during sensitization in allergic contact dermatitis (ACD) are not known.

Objective: Here, we investigated the effect of a strong sensitizer, 2,4-dinitrofluorobenzene (DNFB) on the activity of MAPKs in a dendritic cell (DC) line generated from fetal mouse skin (FSDC), and the results were correlated with the expression of a costimulatory molecule upregulated upon DC maturation, CD40.

Methods: Phosphorylation of ERK1/2 (pERK1/2) and p38 MAPK (pp38 MAPK), and CD40 protein levels, were determined by Western blot. Cellular localization of pERK1/2 and pp38 MAPK were determined by immunocytochemistry using phosphospecific antibodies.

Results: Although with different kinetics, DNFB activated ERK $1 / 2$ and p 38 MAPK, and induced the translocation of the phosphorylated forms of the kinases to the nucleus. In addition, DNFB upregulated significantly CD40 protein levels in FSDC. However, 2,4dichloronitrobenzene (DCNB), an inactive analogue of DNFB, did not affect significantly the phosphorylation of MAPKs and CD40 protein levels. SB203580 and SB202190, inhibitors of the p38 MAPK activity, inhibited DNFB-induced CD40 upregulation, although this effect did not reach statistical significance. In contrast, PD 98059 and U0126, inhibitors of mitogen or extracellular signal-regulated kinase (MEK), had no effect on the CD40 upregulation induced by DNFB.
\end{abstract}

\footnotetext{
* Corresponding author. Tel.: +351239 480209; fax: +351239480208.

E-mail address: cbduarte@ci.uc.pt (C.B. Duarte).
} 
Conclusions: Taken together, these results indicate that the strong sensitizer DNFB activates ERK1/2 and p38 MAPK signaling pathways, and upregulates CD40 protein levels. However, MAPKs do not play a major role in the induction of $C D 40$, one of the phenotypic markers of DC maturation.

(C) 2005 Japanese Society for Investigative Dermatology. Published by Elsevier Ireland Ltd. All rights reserved.

\section{Introduction}

Allergic contact dermatitis (ACD), one of the most common inflammatory skin disorders $[1,2]$, is a delayed-type hypersensitivity reaction caused by a wide range of low molecular weight reactive chemicals (haptens). These haptens enter the skin and bind self-proteins, converting them into immunogenic peptides that are captured and processed by dendritic cells (DCs) [1,3-5]. Skin DC, when activated by inflammatory stimuli or by haptens loose their antigen uptake and processing capacity and acquire professional antigen presenting capacity [1]. For this process, DCs switch chemokine receptors and cytokine synthesis, and upregulate the expression of major histocompability complex (MHC) molecules, and adhesion and costimulatory molecules (CD80, CD86, CD83, CD40) [6-10].

Several skin sensitizers have been shown to upregulate DC expression of CD40, namely 2,4,6-trinitrobenzene sulfonic acid (TNBS), aminophenol, chlorpromazine hydrochloride, dinitrochlorobenzene (DNCB) and the metal allergen nickel sulfate $[11,12]$. CD40, a member of the tumor necrosis factor receptor (TNFR) family, is a $45-50 \mathrm{kDa}$ receptor expressed on a wide range of cell types, including $B$ cells, macrophages and DCs $[13,14]$. During antigen presentation, the interaction of CD40 in DCs with its ligand (CD40L) on activated Tcells is critical for DC maturation and induces Interleukin-12 (IL-12) production, leading to differentiation of T cells into T helper type1 (Th1) $[15,16]$.

The exact cellular and molecular mechanisms of DC activation by haptens remain unclear. An increased phosphorylation of tyrosine residues in murine LCs and human MHC class II positive antigen presenting cells (APC) has been detected upon stimulation with contact sensitizers $[17,18]$, suggesting that activation of protein tyrosine kinases is involved in contact sensitization. Enzymes belonging to the family of MAPKs are strong candidates for activating effector proteins, since they propagate signals generated from different stimuli and have a multiplicity of signal transducing functions, converting extracellular signals into intracellular responses. Three major genetically distinct MAPK pathways are known at present in mammals: the extracellular signal-regulated kinases (ERKs), c-Jun
$\mathrm{NH}_{2}$-terminal kinases (JNKs) and the p38 high osmolarity glycerol protein kinase (p38 MAPKs). Each MAPK is positioned at the bottom of a distinct kinase pathway composed of three sequential dual specific kinases [19-23]. Activated MAPKs can translocate into the nucleus where they may phosphorylate substrates such as transcription factors [22,2427]. TNF $\alpha$ and LPS activate p38 MAPK in DCs [28], and Chemical sensitizers also increase p38 MAPK activity, or p38 MAPK and ERK1/2 activity, in human monocyte derived DCs [29-31] and in a BC1 cell line (an immature DC cell line) [32].

In order to clarify some of the signaling events involved in the sensitization phase of $A C D$ and in DC maturation, we investigated MAPK activation and their putative role in CD40 expression, upon sensitization of a fetal skin dendritic cell line (FSDC) representative of early DC precursors [33]. The effect of a strong sensitizer, 2,4-dinitrofluorobenzene (DNFB), and its inactive analogue, 2,4-dicloronitrobenzene (DCNB), were compared.

\section{Materials and methods}

\subsection{Reagents}

Trypsin was purchased from Invitrogen GIBCO (Paisley, UK). Fetal calf serum was from Biochrom (Berlin, Germany). The 2,4-dinitrofluorobenzene (DNFB) and 2,4-dichloronitrofluorobenzene (DCNB) were obtained from Aldrich (Madrid, Spain). Antibodies against phospho-ERK $1 / 2$ and phospho-JNK were obtained from Promega (Madison, WI). The anti-phospho-p38 MAPK antibody was from Cell Signaling Technology (Beverly, MA). Anti-CD40 antibody was purchased from R\&D Systems (Minneapolis, $M N$ ). The alkaline phosphatase linked goat antirabbit IgG $(\mathrm{H}+\mathrm{L})$ antibody and the ECF substrate were purchased from Amersham Biosciences (Carnaxide, Portugal) and the alkaline phosphatase linked goat anti-rat IgG $(\mathrm{H}+\mathrm{L})$ antibody was purchased from Chemicon (Temecula, CA). Alexa 488conjugated goat anti-rabbit antibody and the Prolong Antifade kit were from Molecular Probes Europe (Leiden, The Netherlands), and the normal goat serum was from Zymed Laboratories (San Francisco, CA). All other reagents were obtained from Sigma 
Chemical Co (Madrid, Spain) or from Merck (Darmstadt, Germany).

\subsection{Cell culture}

The fetal mouse skin dendritic cell line (FSDC), established from fetal mouse skin suspensions [33], was kindly supplied by Dr. G. Girolomoni. The cells were maintained at $37^{\circ} \mathrm{C}$, in a humidified atmosphere at $95 \%$ air and $5 \% \mathrm{CO}_{2}$, in Iscove modified Dulbecco's medium (Sigma) supplemented with 10\% heat inactivated fetal calf serum, $36 \mathrm{mM}$ sodium bicarbonate, $64.4 \mathrm{mM}$ glutamine, $100 \mathrm{U} / \mathrm{ml}$ penicillin and $100 \mu \mathrm{g} / \mathrm{ml}$ streptomycin. Cells were fed with fresh medium every $2-3$ days.

\subsection{Stimulation with chemicals}

Cells were plated at $0.69 \times 10^{6}$ cells/well, in 6-well microplates, for Western blot analysis, and at $0.4 \times 10^{6}$ cells/well, on glass coverslips, for immunocytochemistry assays, and were grown for 2 days. The cells were then placed in serum-free Iscove modified Dulbecco's medium (IMDM) for 10$30 \mathrm{~min}$ before addition of the strong sensitizer DNFB, at $5 \mu \mathrm{g} / \mathrm{ml}$. Cells were then kept at $37^{\circ} \mathrm{C}$ for different periods of time, as indicated in the figure captions. SB203580 $(20 \mu M)$ or SB202190 $(10 \mu \mathrm{M})$ were used as inhibitors of p38 MAPK, and U0126 $(5 \mu \mathrm{M})$ and PD $98059(40 \mu \mathrm{M})$ as inhibitors of the ERK $1 / 2$ pathway. In this case, cells were preincubated with the inhibitors for $1 \mathrm{~h}$ before stimulation with the sensitizer DNFB for $2 \mathrm{~h}$.

\subsection{Cell lysate preparation}

In order to prepare cell extracts for Western blot analysis, after stimulation (as described above) the cells were washed with ice-cold PBS and scraped into ice-cold lysis buffer containing protease and phosphatase inhibitors (50 mM Hepes pH 7.5, $1 \%$ Triton X-100, 100 mM NaCl, 2 mM EGTA, 2 mM EDTA, $2 \mathrm{mM} \mathrm{Na}_{3} \mathrm{VO}_{4}$, and freshly added $1 \mathrm{mM} \mathrm{PMSF}, 2.5 \mu \mathrm{g} /$ $\mathrm{ml}$ Pepstatin, $1 \mathrm{mM}$ DTT, $10 \mu \mathrm{M}$ E64, $20 \mu \mathrm{g} / \mathrm{ml}$ Benzamidin, $10 \mathrm{mM} \mathrm{NaF}$ ). Cell lysates were clarified by centrifugation at $9700 \times \mathrm{g}$, for $30 \mathrm{~min}$, at $4{ }^{\circ} \mathrm{C}$. The supernatants were stored at $-80^{\circ} \mathrm{C}$ until protein determination using the BCA protein assay method (Pierce, Rockford, IL). Cell lysates were then boiled for $5 \mathrm{~min}$ in $6 \times$ sample buffer $(187.5 \mathrm{mM}$ Tris $-\mathrm{HCl} \mathrm{pH}$ 6.8; $12 \%$ w/v SDS; $60 \%$ sucrose; $0.06 \%$ bromophenol Blue and $37.5 \% \beta$-mercaptoethanol) and either used immediately for SDS/PAGE electrophoresis or frozen at $-20{ }^{\circ} \mathrm{C}$ until use. Cell lysates for anti-CD40 Western blots were prepared with the same solutions but without the reducing agent, DTT.

\subsection{Western blot analysis}

Western blot was performed for the analysis of ERK, JNK and p38 MAPK activation (using antibodies specific for the phosphorylated form of the three kinases) and for the analysis of CD40 protein levels. Briefly, equivalent amounts of protein $(25-40 \mu \mathrm{g})$ were loaded onto a $10 \%$ SDS-polyacrylamide gel, subjected to electrophoresis, and transferred to polyvinylidene difluoride (PVDF) membranes (Amersham Biosciences). The membranes were saturated with $5 \%(\mathrm{w} / \mathrm{v})$ fat-free dry milk in Tris-buffered saline $(50 \mathrm{mM}$ Tris, $\mathrm{pH} 8.0,150 \mathrm{mM} \mathrm{NaCl})$ with $0.1 \%$ Tween 20 (TBS-T), for $90 \mathrm{~min}$, at room temperature, or overnight at $4{ }^{\circ} \mathrm{C}$ (for JNK). Blots were then incubated overnight with primary antibodies at $1: 1000$ or $1: 5000$ dilution, for phospho-p38 MAPK and -ERK, respectively. Incubation with the antibodies against CD40 (1:500) and phospho-JNK (1:5000) was performed for $2 \mathrm{~h}$, at room temperature. The solutions of primary antibodies were prepared in $1 \%$ fat free dry milk in TBS-T. After extensive washing with $0.5 \%$ fat free dry milk in TBS-T solution, blots were further incubated for $1 \mathrm{~h}$ at room temperature with goat anti-rabbit or anti-rat IgG antibodies coupled to alkaline phosphatase, at $1: 20,000$ or $1: 10,000$ dilution, respectively, in $1 \%$ fat free dry milk in TBS-T. Blots were then washed in $0.5 \%$ fat free dry milk in TBS-T. The immune complexes were detected using the ECF system (Amersham Biosciences), and the membranes were then scanned with Blue excited fluorescence on the Storm 860 (Amersham Biosciences) according to the manufacturers instructions. The signals were analyzed using the ImageQuant software (Amersham Biosciences).

\subsection{Immunocytochemistry assays}

FSDCs, stimulated as described above, were rinsed with PBS and fixed and permeabilized with methanol:acetone (1:1), for 10 min (for ERK $1 / 2$ detection), or with methanol, for 5 min (for p38 MAPK detection), at $-20^{\circ} \mathrm{C}$. Non-specific binding was blocked by incubation with $20 \%$ (or $10 \%$ for phospho-p38 MAPK) normal goat serum in PBS, for $1 \mathrm{~h}$ at room temperature. Coverslips were then incubated overnight at $4{ }^{\circ} \mathrm{C}$ with the primary antibody (antiphospho-ERK1/2, diluted 1:100, or anti-phospho-p38 MAPK, diluted 1:200) in PBS supplemented with $5 \%$ (or 1.5\% for phospho-p38 MAPK) normal goat serum. The cells were then rinsed, for $15 \mathrm{~min}$, in six changes of PBS, and incubated, for $90 \mathrm{~min}$, at room temperature, with the secondary antibody (Alexa 488-conjugated goat anti-rabbit antibody, diluted $1: 2000$ ) in PBS supplemented with $1 \%$ (or $1.5 \%$ for p38 MAPK) normal goat serum. After rinsing the coverslips as 
(A)
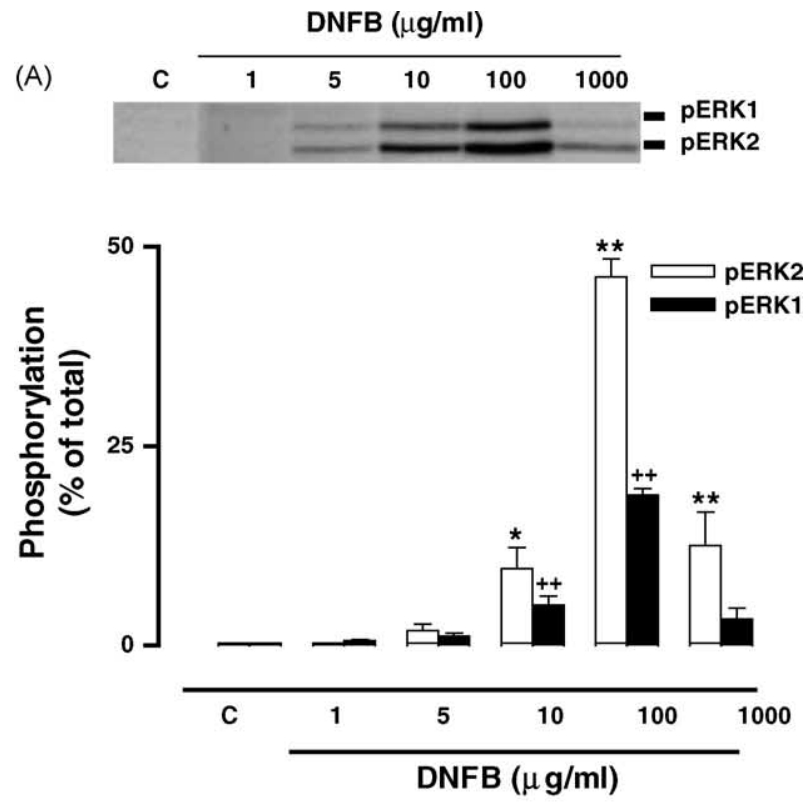

(B)
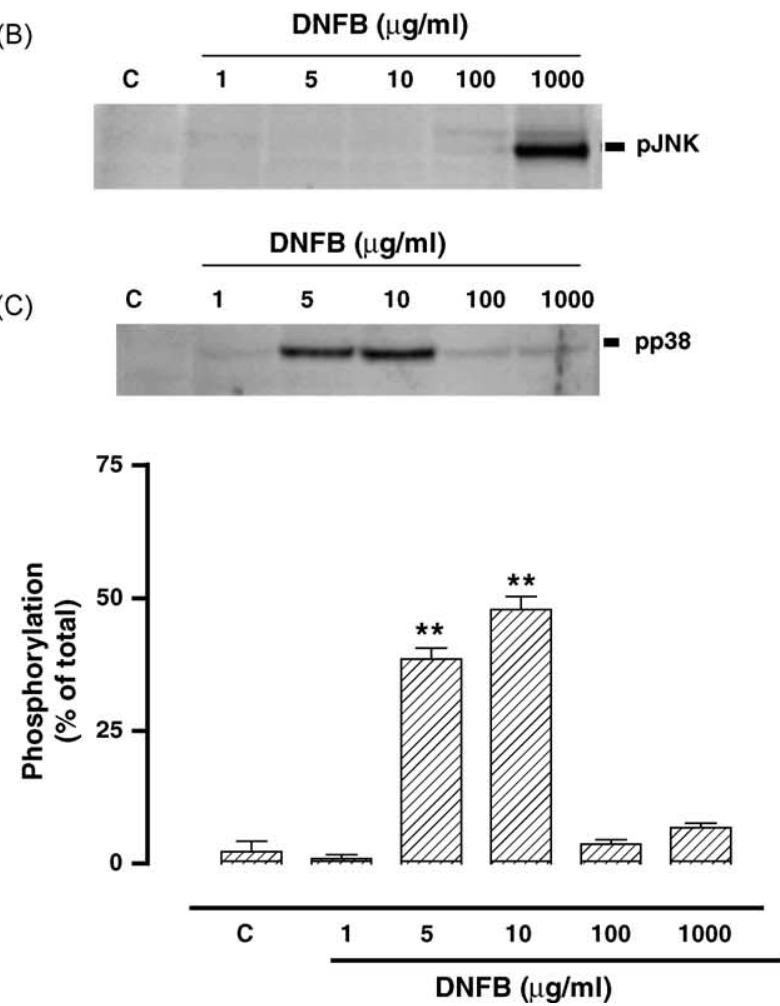

Fig. 1 DNFB induces a dose-dependent phosphorylation of ERK, JNK and p38 MAPK in FSDC cells. Cells were stimulated or not with DNFB, at the indicated concentrations, during $30 \mathrm{~min}$. Equal amounts of protein were loaded on $10 \%$ SDS-polyacrylamide gels and subjected to electrophoresis and electrotransferred to PVDF membranes, before probing with phospho-specific anti-ERK1/2 (A), anti-JNK (B) or anti-p38 MAPK (C) antibodies, as described in Section 2. The results were quantified by scanning the membrane with a fluorescence scanner and analyzed using the ImageQuant software. The results were expressed as the \% of phosphorylation relatively to total in before, they were mounted onto a slide with the Prolong antifade kit to reduce the photobleaching of the fluorescent dye. Negative control experiments were done, consisting on processing the same preparation as described, except for the omission of the primary antibody, and resulted in no non-specific staining. Fluorescent labeling was visualized by confocal microscopy, using a MRC600 confocal system (BIORAD Laboratories, Milan, Italy) linked to a Nikon Optiphot-2 fluorescence microscope, using a $60 \times$ immersion oil objective. A Krypton/Argon mixed laser was used in combination with a fluorescein filter. Image processing included a Kadman filter, using confocal assistant software.

\subsection{Data analysis}

The results are expressed as mean \pm SEM of the indicated number of independent experiments, and statistical analysis was performed using the One-Way ANOVA test, with a Dunnett's post-test or the Repeated measures ANOVA with a Bonferroni's post-test, as indicated. A difference with $p$ value $<0.05$ was considered statistically significant.

\section{Results}

\subsection{Differential effect of DNFB on the activation of MAPKs}

In order to access which signal transduction pathways are activated by DNFB, we examined the content on phosphorylated ERK1 (p44)/ERK2 (p42), p38 MAPK and JNK in FSDC stimulated with different concentrations of DNFB $(1-1000 \mu \mathrm{g} / \mathrm{ml})$, using antibodies raised against the dually phosphorylated (active) form of the enzymes. High doses of DNFB $(1 \mathrm{mg} / \mathrm{ml})$ induced phosphorylation of the three MAPKs (Fig. 1). However, for lower doses of DNFB, only ERK $1 / 2$ and p38 MAPK were found to be phosphorylated. The maximal effect on ERK1/2 phosphorylation was observed for $100 \mu \mathrm{g} / \mathrm{ml}$ (Fig. 1A), whereas DNFB induced a strong phosphorylation of p38 MAPK only at 5 or $10 \mu \mathrm{g} / \mathrm{ml}$ (Fig. 1C). Since DNFB at concentrations higher than $5 \mu \mathrm{g} / \mathrm{ml}$ reduce the viability of the cells as evaluated by the MTT assay [34] (data not shown), all subsequent studies were performed using $5 \mu \mathrm{g} / \mathrm{ml}$ of DNFB.

each experiment. Data are expressed as the mean \pm SEM of three independent experiments. Statistical significance was calculated by the One-Way ANOVA test with a Dunnett's post-test ${ }^{*} p<0.05 ;{ }^{* *} p<0.01$ as compared to the control; ${ }^{++} p<0.01$ as compared to the control ERK1 phosphorylation (A)). 


\subsection{ERK1/2 and p38 MAPK are activated by DNFB with different kinetics}

The time course of ERK1/2 and p38 MAPK phosphorylation was determined in order to investigate

(A)
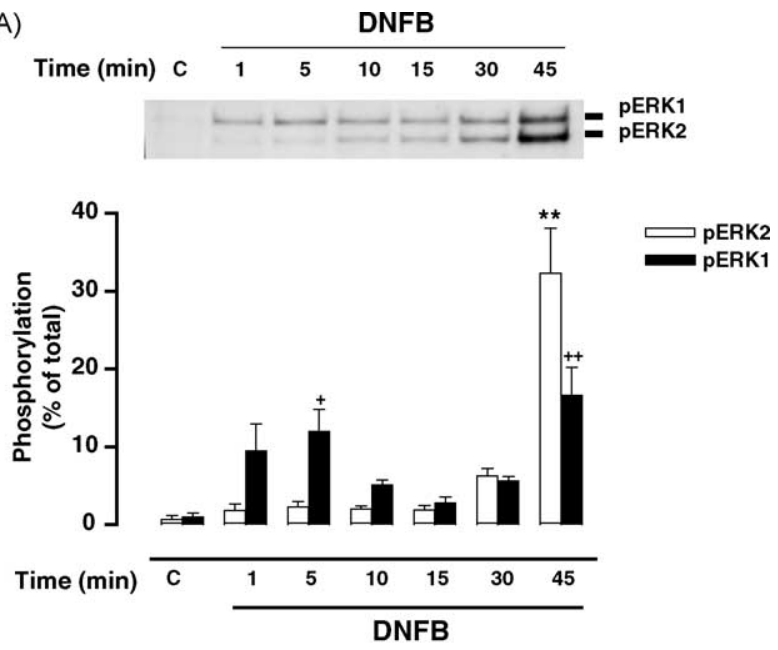

(B)
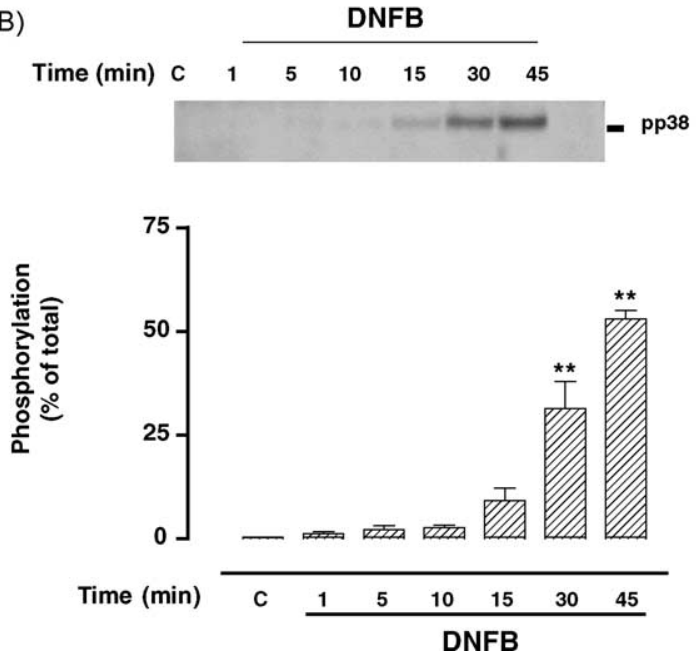

Fig. 2 DNFB induces a time-dependent increase of ERK1/2 and p38 MAPK phosphorylation in FSDC cells. Cells were stimulated, or not, with $5 \mu \mathrm{g} / \mathrm{ml}$ of DNFB and the cell lysates were collected at the indicated times after stimulation. Equal amounts of protein were loaded on $10 \%$ SDS-polyacrylamide gels, subjected to electrophoresis and electrotransferred to PVDF membranes, before probing with a phospho-specific anti-ERK1/2 antibody (A) or anti-p38 MAPK antibody (B), as described in Section 2. The results were quantified by scanning the membrane with a fluorescence scanner and analyzed using the ImageQuant software. The results were expressed as the \% of phosphorylation relatively to total in each experiment. Data are expressed as the mean \pm SEM of four independent experiments. Statistical significance was calculated by the One-Way ANOVA test with a Dunnett's post-test $\left({ }^{*} p<0.05 ;{ }^{* *} p<0.01\right.$ as compared to the control; ${ }^{+} p<0.05,{ }^{++} p<0.01$ as compared to the control ERK1 phosphorylation $(A))$. whether the activation of MAPKs by DNFB occurred with similar kinetics. FSDC cells were stimulated with DNFB for different periods of time and phosphorylation of the MAPKs was examined by Western blot analysis (Fig. 2). ERK1/2 phosphorylation (activation) was detected as soon as $1 \mathrm{~min}$ after exposure to DNFB (Fig. 2A). The ERK1 (44 kDa) activation induced by DNFB was biphasic, with a rapid and transient phosphorylation (activation) that peaked around 1-5 min, followed by a second phase of kinase activity at $30-45 \mathrm{~min}$. The kinetics of ERK2 (42 kDa) phosphorylation followed a distinct pattern. After the first minute of stimulation, ERK2 phosphorylation showed a slight increase relatively to the control, which however did not reach statistical significance, in contrast with the results obtained for ERK1. The delayed response (45 min) was similar for both isoforms of ERK, but ERK2 phosphorylation was much stronger.

p38 MAPK showed a slower kinetics of phosphorylation, starting at $15 \mathrm{~min}$ of stimulation and reaching the maximal increase within $30-45 \mathrm{~min}$ of stimulation with DNFB (Fig. 2B). Taken together, our results indicate that both ERK $1 / 2$ and p38 MAPK are phosphorylated upon DNFB stimulation, although with different kinetics.

The specificity of the sensitizing effect of DNFB was tested using its structurally related analogue DCNB, which has no [35] or very low [36] skin sensitization potential. In contrast with the results observed with DNFB, DCNB did not affect the phosphorylation of ERK1/2 or p38 MAPK (Fig. 3A and B). Therefore, our data indicate that the effect observed with DNFB is specific of its allergenic property.

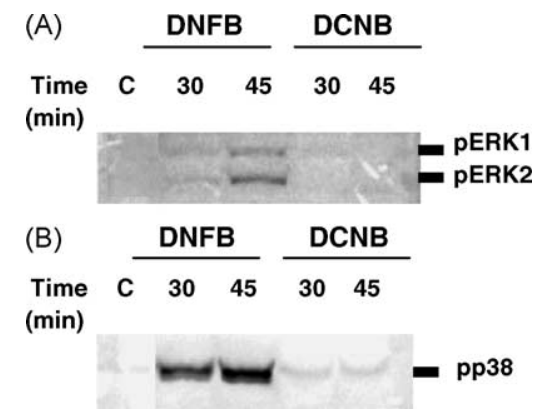

Fig. 3 DCNB, the inactive analogue of DNFB, does not affect ERK1/2 and p38 MAPK phosphorylation. Cells were stimulated or not with $5 \mu \mathrm{g} / \mathrm{ml}$ of DCNB or DNFB and the cell lysates were collected at the indicated times after stimulation. Equal amounts of protein were loaded on 10\% SDSpolyacrylamide gels, subjected to electrophoresis and electrotransferred to PVDF membranes, before probing with a phospho-specific anti-ERK1/2 antibody (A) or antip38 MAPK antibody (B), as described in Section 2. Data are representative of three independent experiments. 


\subsection{DNFB-induced nuclear translocation of activated ERK 1/2 and p38 MAPK}

Since the phosphorylation of MAPKs is frequently associated with their translocation to the nucleus
[25], immunocytochemistry experiments were performed to determine the effect of DNFB on their subcellular location (Fig. 4). Control cells, not incubated with DNFB, showed low immunoreactivity with the phospho-specific anti-active ERK1/2 anti-

\section{(A) PERK $1 / 2$}
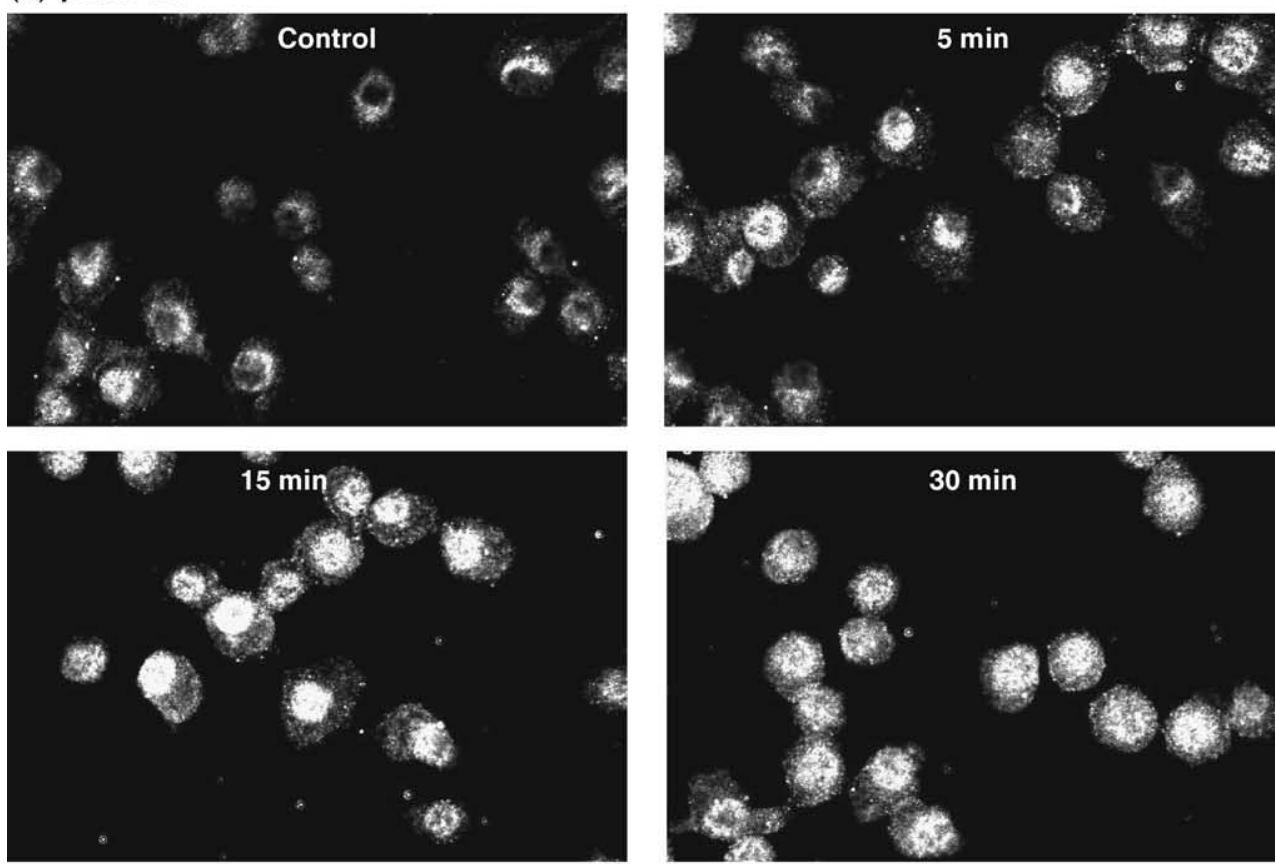

(B) $\mathrm{pp38}$
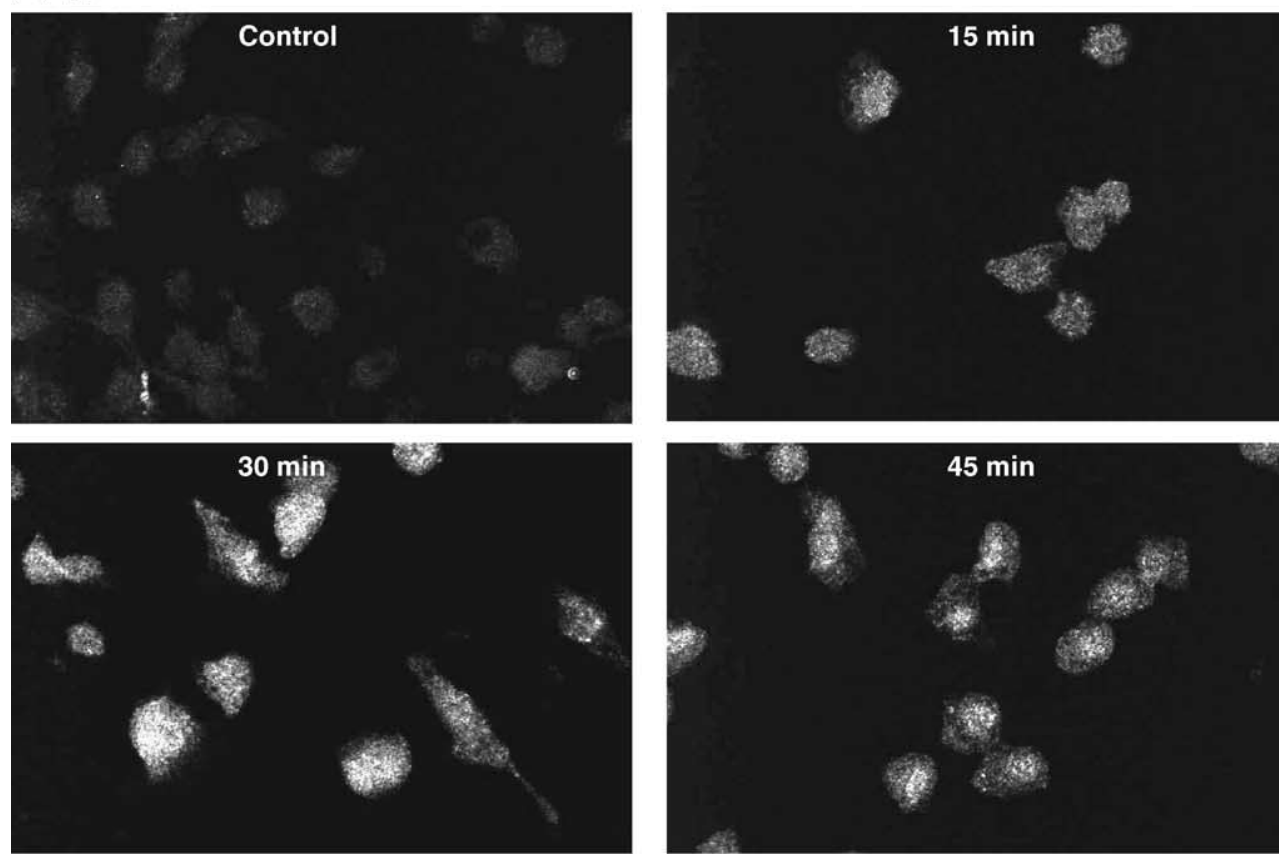

Fig. 4 DNFB-induced activation and translocation of ERK1/2 and p38 MAPK to the nucleus. The cells were stimulated or not with DNFB $(5 \mu \mathrm{g} / \mathrm{ml})$, and immunostained with phospho-specific anti-ERK1/2 (A) or anti-p38 MAPK (B) antibodies, as described in Section 2. The figures are representative of the results obtained in at least three independent experiments. 
body (Fig. 4A), indicating that there is a minor resting activity of ERK $1 / 2$ in the cytosol. Translocation of pERK $1 / 2$ to the nucleus was observed after 5 min of stimulation with DNFB and was maintained over $15 \mathrm{~min}$. After $30 \mathrm{~min}$ of stimulation p-ERK1/2 was still found in the cytosol. In contrast, DNFB increased the amount of phosphorylated p38 MAPK in the nucleus only after $30 \mathrm{~min}$ of stimulation (Fig. 4B). These results demonstrate that in FSDC the two MAPK pathways are activated by DNFB, although with a different time course. Their translocation to the nucleus, confirmed by these observations, suggests that these signaling molecules may be involved in the regulation of nuclear proteins by DNFB, namely transcription factors.

\subsection{DNFB-induced an increase of CD40 immunoreactivity}

Protein levels of CD40, a surface marker related with the maturation and activation of $D C$, were measured after stimulation of FSDC with DNFB. Using an antibody that recognizes CD40, we observed that CD40 protein levels are low in resting FSDC, but increase significantly upon stimulation with DNFB (Fig. 5). This increase was detected after 30 min of stimulation (2.5-fold above control) and further increased until $3-8 \mathrm{~h}$ of incubation with the sensitizer. Similar results were obtained when the cells were stimulated with DNFB in culture medium containing serum (not shown). In contrast with the results obtained with DNFB, CD40 protein levels were not affected by DCNB (Fig. 5).

\subsection{DNFB-induced CD40 immunoreactivity is partially dependent on the activation of p38 MAPK}

In order to investigate the role of ERK1/2 and p38 MAPK pathways on the activation and maturation of FSDC, we studied the effect of MEK and p38 MAPK inhibitors on the expression of CD40. The effect of DNFB on CD40 protein levels was not changed in the presence of the MEK inhibitors PD098059 and U0126 [37,38] (Fig. 6A). In contrast, the p38 MAPK inhibitors, SB203580 [39] and SB202190 [37], partly reduced the effect of the sensitizer on CD40 protein levels after $2 \mathrm{~h}$ of stimulation (Fig. 6B). Although the effect was very consistent in all the experiments performed the inhibition of DNFB-induced CD40 upregulation did not reach statistical significance. These results suggest that activation of p38 MAPK, but not ERK1/2, is involved in the upregulation of CD40 protein levels induced by DNFB in FSDC, but it is not the main signaling pathway involved.

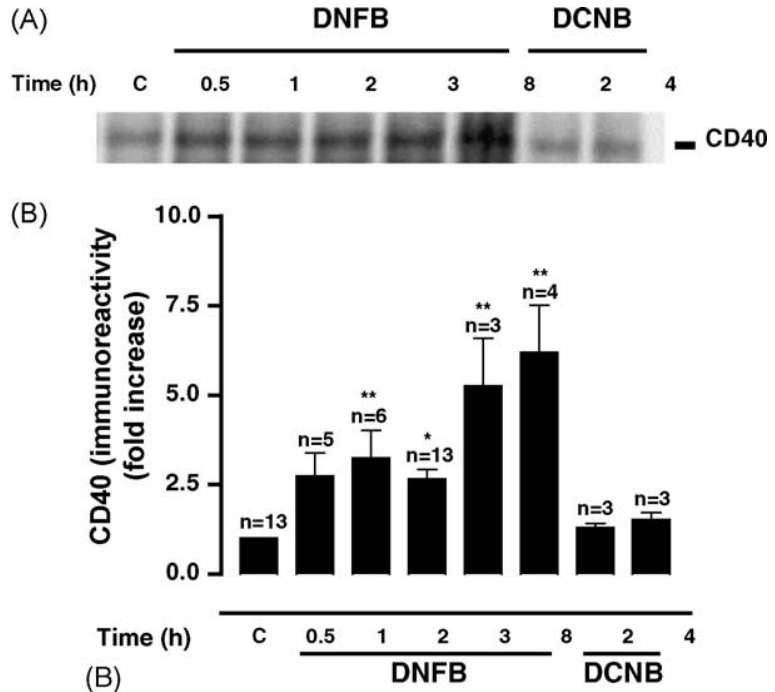

Fig. 5 DNFB, but not DCNB, increases CD40 protein levels in FSDC. Cells were stimulated or not with $5 \mu \mathrm{g} /$ $\mathrm{ml}$ DNFB or DCNB. Cell lysates were collected at the indicated times after stimulation. Equal amounts of protein were loaded on $10 \%$ SDS-polyacrylamide gels, subjected to electrophoresis and electrotransferred to PVDF membranes, before probing with an anti-CD40 antibody (A). The results were quantified by scanning the membrane with a fluorescence scanner and analyzed using the ImageQuant software. The results were expressed as fold increase relatively to the control in each experiment (B). Data are expressed as the mean \pm SEM of four independent experiments. Statistical significance was calculated by the One-Way ANOVA test with a Dunnett's post-test ( ${ }^{*} p<0.05 ;{ }^{* *} p<0.01$ as compared to the control).

\section{Discussion}

The major cellular events involved in the maturation and migration of DC to lymph nodes during the process of skin sensitization are still unknown. In the present work, we show that while high doses of the chemical sensitizer DNFB induced the activation of the three MAPK family members, ERK, JNK and p38 MAPK, only ERK $1 / 2$ and $\mathrm{p} 38$ MAPK were activated in a skin-derived dendritic cell line (FSDC) by subtoxic concentrations of the sensitizer. Furthermore, DNFB also increased CD40 protein levels in FSDC by a mechanism largely independent of the MAPK signaling pathways.

ERKs are found in different subcellular compartments, phosphorylating a wide range of proteins, from the plasma membrane to the nucleus. These proteins of the MAPK family have been shown to play important roles in cell growth and differentiation $[20,23]$, cell survival and in inflammatory responses $[22,40-42]$. In the present study, we found that DNFB has a biphasic effect on ERK1/2 activity in the cells: a rapid activation of ERK1 (within 1 min) 
(A)
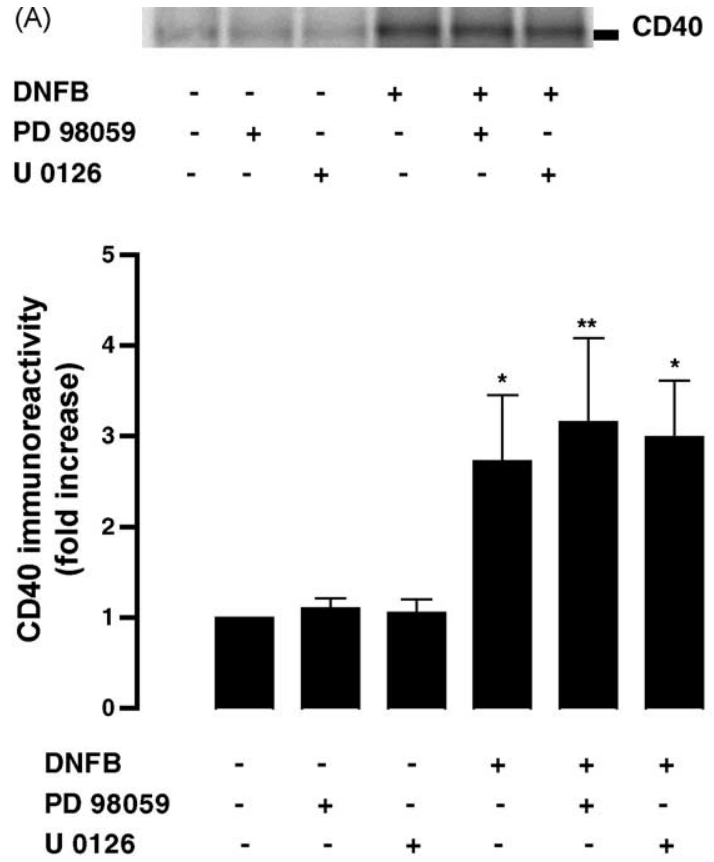

(B)
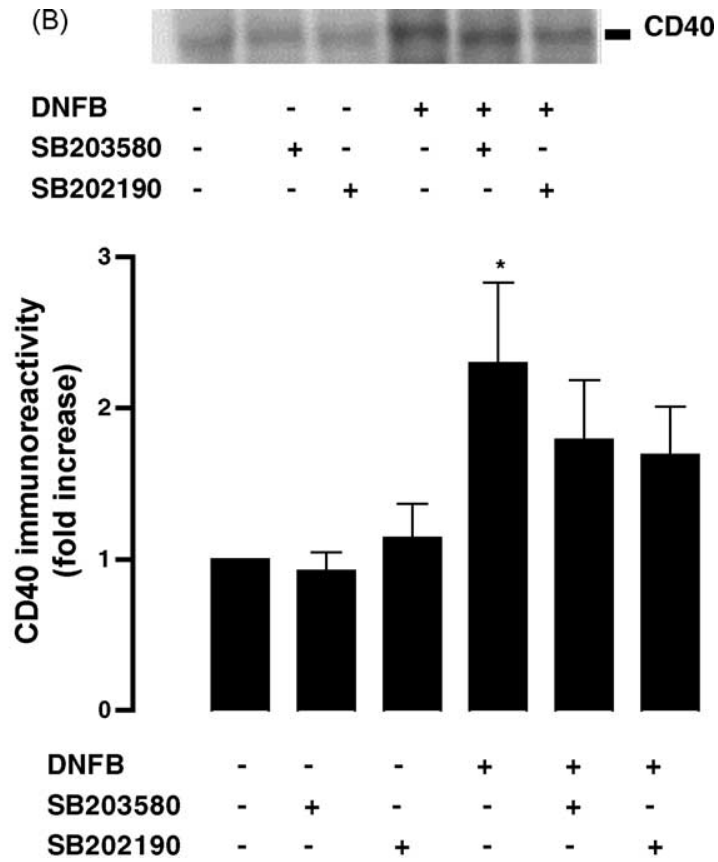

Fig. 6 p38 MAPK, but not ERK, is partially involved in DNFB induced increase of CD40 protein levels in FSDC. To access the involvement of ERK $1 / 2$ or p38 MAPK in the increase of CD40 protein levels, cells were stimulated or not with $5 \mu \mathrm{g} / \mathrm{ml}$ DNFB in the presence of MEK inhibitors (PD $98059(40 \mu \mathrm{M})$ or U0126 $(5 \mu \mathrm{M}))(\mathrm{A})$ or in the presence of p38 MAPK inhibitors (SB203580 (20 $\mu \mathrm{M})$ or SB202190 (10 $\mu \mathrm{M}))(\mathrm{B})$, and collected after $2 \mathrm{~h}$ of stimulation. Equal amounts of protein were loaded on $10 \%$ SDS-polyacrylamide gels, subjected to electrophoresis and electrotransferred to PVDF membranes, before probing with an anti-CD40 antibody. The results were quantified by scanning the membrane with a fluorescence scanner and analyzed using the ImageQuant software. The results were expressed as fold increase relatively to the control in each experiment. Data are expressed as the mean \pm SEM of four independent experiments. Statistical significance was calculated by the Repeated measures ANOVA test with a Bonferroni's post-test $\left({ }^{*} p<0.05 ;{ }^{* *} p<0.01\right.$ as compared to the control).

and a further increase until 5 min of stimulation (Fig. 2A), which occurred in parallel with ERK translocation to the nucleus in FSDC (Fig. 4A). Once in the nucleus ERK1/2 are likely to be involved in the regulation of transcription factors [27,43], namely $\mathrm{NF}-\kappa \mathrm{B}$, that we have previously shown to be activated by DNFB at $15 \mathrm{~min}$ in these cells [44]. The nuclear translocation of the phosphorylated ERK1/2 is in agreement with previous work performed in other cell types $[22,25]$. In a second phase, a delayed increase in ERK1 activity was observed after 30-45 min stimulation. This second increase in ERK1 activity was accompanied by an increase in ERK2 activity (Fig. 2A), and was characterized by a strong immunoreactivity in the cytoplasm (Fig. 4A). The difference observed in the activation of the two ERK isoforms suggests that they may mediate different processes.

Previous studies using LPS, an immunostimulatory molecule which also induces the maturation of DCs by binding to receptor molecules at the cell surface, also showed activation of ERK1/2 in macrophages and DCs, although with a different kinetic $[42,45]$. In the latter cell type the activation of ERK $1 / 2$ by LPS was involved in the regulation of cell survival [42]. Additionally, in murine B cells it was further demonstrated that stimulation with two distinct agents (CD40 and IgM) differentially regulates ERK subcellular localization, supporting the notion that ERK could mediate different effector functions in these cells upon stimulation with different agents [46]. Therefore, the differential activation of ERK1 and ERK2 in FSDC stimulated with DNFB may also cause distinct functional responses in the cell.

p38 MAPK, together with JNK, are considered stress activated kinases, and p38 MAPK has also been implicated in the inflammatory response $[20,22,26,47]$. Here we observed that DNFB induced activation of p38 MAPK after a lag-phase of about $15 \mathrm{~min}$, and the activity of the kinase further increased until $45 \mathrm{~min}$ of incubation (Fig. 2B). Furthermore, the phosphorylated form of p38 MAPK was translocated to the nucleus in a significant number of cells after 30 min stimulation with DNFB, as determined by immunocytochemistry (Fig. 4B). Our findings are in agreement with recent reports 
showing p38 MAPK phosphorylation in human monocyte-derived dendritic cells stimulated with DNFB [30] or 2,4-dinitrochlorobenzene (DNCB, which has a similar sensitizing potential as DNFB) [29]. However, in the former study the activation of p38 MAPK was observed for shorter incubation periods. Furthermore, we also detected a strong activation of ERK1/ 2 by DNFB, which was not observed by Arrighi et al. [30]. In experiments using human monocyte-derived DCs these two signaling pathways were also shown to be activated by another skin sensitizer $-\mathrm{NiCl}_{2}-$ [29], which, as we have shown for DNFB [44], also activates the NF-кB transcription factor [29]. The activation of p38 MAPK by DNCB was also recently observed using an immature murine dendritic cell line (BC1 cells) [32]. In contrast, and in agreement with other reports $[29,30]$, we observed that a nonsensitizer, DCNB (an inactive analogue of DNFB), did not activate any of the MAPKs.

Several reports using immunostimulatory molecules, such as LPS, that also promotes DC maturation, also described an increase of p38 MAPK and ERK in dendritic cells $[28-30,48]$. Also, using a p38 MAPK inhibitor, we have previously shown that this pathway is involved in NO production induced by LPS in FSDC [49]. Taken together, these findings suggest that the signaling pathways described here are shared by chemical sensitizers and immunostimulatory molecules.

We further studied the effect of DNFB on the expression of CD40, a membrane-associated receptor that is upregulated in DCs during maturation induced by several stimuli, including skin sensitizers. The results show that CD40 protein levels are upregulated in FSDC as soon as 30 min after DNFB stimulation and further increased during the time of the experiment, whereas the non-sensitizer DCNB had no effect. In preliminary experiments we found that DNFB also slightly upregulated CD80 and CD86 protein levels, but the effects were not as significant as those obtained for CD40 (unpublished observations).

ERK and, specially, p38 MAPK activation, seem to be involved in the maturation of DCs, namely in increasing the expression of costimulatory molecules $[28-30,48]$. However, in the present work we observed that only p38 MAPK inhibitors showed a slight effect on DNFB-induced upregulation of $\mathrm{CD} 40$, suggesting that other signaling pathways are also involved. p38 MAPK was shown to be involved in CD40 upregulation induced by LPS [30] and by plasmin [50], but a role for p38 MAPK in CD40 upregulation induced by skin sensitizers had not been described. In our work, the decrease in CD40 protein levels detected in the presence of p38 MAPK inhibitors was not statistically significant, indicating that this kinase is not the main signaling pathway involved in CD40 upregulation induced by DNFB in FSDC.

Although in FSDC stimulated with DNFB we detected both the activation of MAPKs in FSDC and an increase in CD40, only p38 MAPK could be slightly correlated with the regulation of CD40 protein levels. Therefore, the role of ERK and p38 MAPK activation in the maturation of FSDC remains to be assessed. The translocation of both ERK $1 / 2$ and p38 MAPK to the nucleus suggests that they are involved in the regulation of transcription factors that may contribute to FSDC maturation. Interestingly, in monocyte-derived DCs stimulated with LPS and TNF- $\alpha$, the ERK pathway has been described as a negative regulator of $D C$ maturation, opposing to the p38 MAPK pathway [51].

In conclusion, DNFB induced the activation of ERK $1 / 2$ and P38 MAPK in FSDC, as well as the upregulation of CD40 expression, whereas its inactive analogue DCNB did not. This indicates that this strong sensitizer induces phenotypical changes that may represent an early activation state of the DCs, which could be responsible for the sensitization phase of ACD. Furthermore, p38 MAPK seems to have a minor role in the upregulation of CD40 expression, suggesting that the MAPKs signaling pathways are involved in other processes that remain to be identified.

Studies of the intracellular signaling pathways used by allergens to activate DC and to promote their presentation to the immune system, and of how pharmacological tools interfere with these pathways, are very relevant to the understanding of the physiopathology of allergic contact dermatitis and its treatment. If data on different skin sensitizers proves to be reproducible, in vitro $D C$ activation can be used to study the sensitization potential of chemicals applied to the skin, instead of using living animals for LLNA.

\section{Acknowledgements}

This work was supported by FCT and FEDER.

\section{References}

[1] Enk AH. Allergic contact dermatitis: understanding the immune response and potential for targeted therapy using cytokines. Mol Med Today 1997;3(10):423-8.

[2] Griem P, Wulferink M, Sachs B, Gonzalez JB, Gleichmann E. Allergic and autoimmune reactions to xenobiotics: how do they arise? Immunol Today 1998;19(3):133-41. 
[3] Grabbe S, Schwarz T. Immunoregulatory mechanisms involved in elicitation of allergic contact hypersensitivity. Immunol Today 1998;19(1):37-44.

[4] Germain RN. Behind the veils: uncovering the biology of dendritic cells. Cell 1999;719-22.

[5] Girolomoni G, Ricciardi-Castagnoli P. Dendritic cells hold promise for immunotherapy. Immunol Today 1997;18(3): 102-4.

[6] Cyster JG. Chemokines and cell migration in secondary lymphoid organs. Science 1999;286(5447):2098-102.

[7] Dieu MC, Vanbervliet B, Vicari A, Bridon JM, Oldham E, AitYahia $S$, et al. Selective recruitment of immature and mature dendritic cells by distinct chemokines expressed in different anatomic sites. J Exp Med 1998;188(2):373-86.

[8] Hart DN. Dendritic cells: unique leukocyte populations which control the primary immune response. Blood 1997;90(9):3245-87.

[9] Sallusto F, Schaerli P, Loetscher P, Schaniel C, Lenig D, Mackay CR, et al. Rapid and coordinated switch in chemokine receptor expression during dendritic cell maturation. Eur J Immunol 1998;28(9):2760-9.

[10] Yamaguchi Y. Developmental regulation by cytokines of bone marrow-derived dendritic cells and epidermal Langerhans cells. Microbiol Immunol 1998;42(9):639-50.

[11] Coutant KD, de Fraissinette AB, Cordier A, Ulrich P. Modulation of the activity of human monocyte-derived dendritic cells by chemical haptens, a metal allergen, and a staphylococcal superantigen. Toxicol Sci 1999;52(2):189-98.

[12] Coutant KD, Ulrich P, Thomas H, Cordier A, Brugerolle de Fraissinette A. Early changes in murine epidermal cell phenotype by contact sensitizers. Toxicol Sci 1999;48(1):74-81.

[13] van Kooten C, Banchereau J. CD40-CD40 ligand. J Leukoc Biol 2000;67(1):2-17.

[14] Tan J, Town T, Mori T, Obregon D, Wu Y, DelleDonne A, et al. CD40 is expressed and functional on neuronal cells. EMBO J 2002;21(4):643-52.

[15] Cella M, Scheidegger D, Palmer-Lehmann K, Lane P, Lanzavecchia $A$, Alber $G$. Ligation of CD40 on dendritic cells triggers production of high levels of interleukin-12 and enhances $T$ cell stimulatory capacity: T-T help via APC activation. J Exp Med 1996;184(2):747-52.

[16] Koch F, Stanzl U, Jennewein P, Janke K, Heufler C, Kampgen $\mathrm{E}$, et al. High level IL-12 production by murine dendritic cells: upregulation via MHC class II and CD40 molecules and downregulation by IL-4 and IL-10. J Exp Med 1996;184(2): 741-6.

[17] Kuhn U, Brand P, Willemsen J, Jonuleit $H$, Enk $A H$, van Brandwijk-Petershans R, et al. Induction of tyrosine phosphorylation in human MHC class II-positive antigen-presenting cells by stimulation with contact sensitizers. J Immunol 1998;160(2):667-73.

[18] Neisius U, Brand P, Plochmann S, Saloga J, Knop J, Becker D. Detection of increased tyrosine phosphorylation in murine Langerhans cells after stimulation with contact sensitizers. Arch Dermatol Res 1999;291(1):22-7.

[19] Cobb MH, Goldsmith EJ. How MAP kinases are regulated. J Biol Chem 1995;270(25):14843-6.

[20] Davis RJ. The mitogen-activated protein kinase signal transduction pathway. J Biol Chem 1993;268(20):14553-6.

[21] Kyriakis JM, Banerjee P, Nikolakaki E, Dai T, Rubie EA, Ahmad $M F$, et al. The stress-activated protein kinase subfamily of cJun kinases. Nature 1994;369(6476):156-60.

[22] Matos TJ, Duarte CB, Carvalho AP, Lopes MC. Activation and physiological roles of the mitogen activated protein kinases (MAPKs). Nato Sci Ser 2001;318(IOS Press):12-28.

[23] Robinson MJ, Cobb MH. Mitogen-activated protein kinase pathways. Curr Opin Cell Biol 1997;9(2):180-6.
[24] Ferrigno P, Posas F, Koepp D, Saito H, Silver PA. Regulated nucleo/cytoplasmic exchange of HOG1 MAPK requires the importin beta homologs NMD5 and XPO1. EMBO J 1998;17(19):5606-14.

[25] Khokhlatchev AV, Canagarajah B, Wilsbacher J, Robinson M, Atkinson M, Goldsmith E, et al. Phosphorylation of the MAP kinase ERK2 promotes its homodimerization and nuclear translocation. Cell 1998;93(4):605-15.

[26] Tibbles LA, Woodgett JR. The stress-activated protein kinase pathways. Cell Mol Life Sci 1999;55(10):1230-54.

[27] Treisman R. Regulation of transcription by MAP kinase cascades. Curr Opin Cell Biol 1996;8(2):205-15.

[28] Ardeshna KM, Pizzey AR, Devereux S, Khwaja A. The PI3 kinase, p38 SAP kinase, and NF-kappaB signal transduction pathways are involved in the survival and maturation of lipopolysaccharide-stimulated human monocyte-derived dendritic cells. Blood 2000;96(3):1039-46.

[29] Aiba S, Manome H, Nakagawa S, Mollah ZU, Mizuashi M, Ohtani T, et al. p38 Mitogen-activated protein kinase and extracellular signal-regulated kinases play distinct roles in the activation of dendritic cells by two representative haptens, $\mathrm{NiCl} 2$ and 2,4-dinitrochlorobenzene. J Invest Dermatol 2003;120(3):390-9.

[30] Arrighi JF, Rebsamen M, Rousset F, Kindler V, Hauser C. A critical role for $\mathrm{p} 38$ mitogen-activated protein kinase in the maturation of human blood-derived dendritic cells induced by lipopolysaccharide, TNF-alpha, and contact sensitizers. J Immunol 2001;166(6):3837-45.

[31] Mollah ZU, Aiba S, Nakagawa S, Hara M, Manome H, Mizuashi $M$, et al. Macrophage colony-stimulating factor in cooperation with transforming growth factor-beta1 induces the differentiation of CD34+ hematopoietic progenitor cells into Langerhans cells under serum-free conditions without granulocyte-macrophage colony-stimulating factor. J Invest Dermatol 2003;120(2):256-65.

[32] lijima N, Yanagawa Y, Onoe K. Role of early- or late-phase activation of p38 mitogen-activated protein kinase induced by tumour necrosis factor-alpha or 2,4-dinitrochlorobenzene during maturation of murine dendritic cells. Immunology 2003;110(3):322-8.

[33] Girolomoni G, Lutz MB, Pastore S, Assmann CU, Cavani A, Ricciardi-Castagnoli P. Establishment of a cell line with features of early dendritic cell precursors from fetal mouse skin. Eur J Immunol 1995;25(8):2163-9.

[34] Cruz MT, Duarte CB, Goncalo M, Figueiredo A, Carvalho AP, Lopes MC. The sensitizer 2,4-dinitrofluorobenzene activates caspase- 3 and induces cell death in a skin dendritic cell line. Int J Toxicol 2003;22(1):43-8.

[35] Enk AH, Katz SI. Early molecular events in the induction phase of contact sensitivity. Proc Natl Acad Sci USA 1992;89(4):1398-402.

[36] Basketter DA, Scholes EW, Fielding I, Dearman RJ, Hilton J, Kimber I. Dichloronitrobenzene: a reappraisal of its skin sensitization potential. Contact Dermatitis 1996;34(1): 55-8.

[37] Davies SP, Reddy H, Caivano M, Cohen P. Specificity and mechanism of action of some commonly used protein kinase inhibitors. Biochem J 2000;351(Pt 1):95-105.

[38] Favata MF, Horiuchi KY, Manos EJ, Daulerio AJ. Identification of a novel inhibitor of mitogen-activated protein kinase. J Biol Chem 1998;273:18623-32.

[39] Cuenda A, Rouse J, Doza YN, Meier R, Cohen P, Gallagher TF, et al. SB 203580 is a specific inhibitor of a MAP kinase homologue which is stimulated by cellular stresses and interleukin-1. FEBS Lett 1995;364(2):229-33.

[40] Bhat NR, Zhang P, Lee JC, Hogan EL. Extracellular signalregulated kinase and p38 subgroups of mitogen- activated 
protein kinases regulate inducible nitric oxide synthase and tumor necrosis factor-alpha gene expression in endotoxinstimulated primary glial cultures. J Neurosci 1998;18(5): 1633-41.

[41] Garcia J, Lemercier B, Roman-Roman S, Rawadi G. A Mycoplasma fermentans-derived synthetic lipopeptide induces AP-1 and NF-kappaB activity and cytokine secretion in macrophages via the activation of mitogen-activated protein kinase pathways. J Biol Chem 1998;273(51):34391-8.

[42] Rescigno M, Martino M, Sutherland CL, Gold MR, RicciardiCastagnoli P. Dendritic cell survival and maturation are regulated by different signaling pathways. J Exp Med 1998;188(11):2175-80.

[43] Hardy K, Chaudhri G. Activation and signal transduction via mitogen-activated protein (MAP) kinases in T lymphocytes. Immunol Cell Biol 1997;75(6):528-45.

[44] Cruz MT, Duarte CB, Goncalo M, Figueiredo A, Carvalho AP, Lopes MC. Differential activation of nuclear factor kappa $B$ subunits in a skin dendritic cell line in response to the strong sensitizer 2,4-dinitrofluorobenzene. Arch Dermatol Res 2002;294(9):419-25.

[45] Weinstein SL, Sanghera JS, Lemke K, DeFranco AL, Pelech SL. Bacterial lipopolysaccharide induces tyrosine phosphorylation and activation of mitogen-activated protein kinases in macrophages. J Biol Chem 1992;267(21):14955-62.
[46] Shirakata Y, Ishii K, Yagita H, Okumura K, Taniguchi M, Takemori T. Distinct subcellular localization and substrate specificity of extracellular signal-regulated kinase in B cells upon stimulation with IgM and CD40. J Immunol 1999;163(12):6589-97.

[47] Herlaar E, Brown Z. p38 MAPK signalling cascades in inflammatory disease. Mol Med Today 1999;5(10):439-47.

[48] An H, Yu Y, Zhang M, Xu H, Qi R, YanX, et al. Involvement of ERK, p38 and NF-kappaB signal transduction in regulation of TLR2, TLR4 and TLR9 gene expression induced by lipopolysaccharide in mouse dendritic cells. Immunology 2002;106(1):38-45.

[49] Cruz MT, Duarte CB, Goncalo M, Carvalho AP, Lopes MC. Involvement of JAK2 and MAPK on type II nitric oxide synthase expression in skin-derived dendritic cells. Am J Physiol 1999;277(6 Pt 1):1050-7.

[50] Burysek L, Syrovets T, Simmet T. The serine protease plasmin triggers expression of MCP-1 and CD40 in human primary monocytes via activation of p38 MAPK and janus kinase (JAK)/STAT signaling pathways. J Biol Chem 2002;277(36): 33509-17.

[51] Puig-Kroger A, Relloso M, Fernandez-Capetillo O, Zubiaga A, Silva A, Bernabeu C, et al. Extracellular signal-regulated protein kinase signaling pathway negatively regulates the phenotypic and functional maturation of monocyte-derived human dendritic cells. Blood 2001;98(7):2175-82.

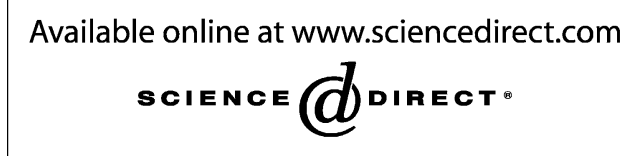

